\title{
Vínculo afectivo con las figuras de apego, calidad en las relaciones románticas y autoconcepto en estudiantes de una universidad privada de Lima Este-Perú
}

\author{
Affective bond with the attachment figures, quality in romantics relationships and self-concept in \\ teenagers of a private university of Lima-Perú
}

Gloria Arias Orezano', Yesenia Ávila Bacilio², Chelita Santillán Mejía ${ }^{3}$

\begin{abstract}
RESUMEN
Objetivo: El objetivo del estudio fue determinar el grado de influencia del vínculo afectivo con las figuras de apego (madre, padre y pares) y la calidad en las relaciones románticas sobre el autoconcepto en 215 estudiantes, de 16 a 20 años, de una universidad privada de Lima. Material y métodos: El diseño fue no experimental, explicativo y de corte transversal. Se utilizaron tres instrumentos: el Inventario de Apego con Padres y Pares (IPPA), la Escala Multidimensional de Autoconcepto Forma 5 (AF5) y la Escala de Calidad en las Relaciones Románticas (NRI). Resultados: Los resultados mostraron que el vínculo afectivo con las figuras de apego y la calidad en relaciones románticas explican, en un 44,7\%, la variabilidad del autoconcepto. Se encontró, además, que el autoconcepto es influenciado por el vínculo afectivo, en un 43,7\%. Asimismo, el autoconcepto familiar es explicado, en un 50,4\%, por el vínculo afectivo; sin embargo, para el autoconcepto social y emocional, el vínculo afectivo con los pares explica un $25,9 \%$ y $18,1 \%$ respectivamente. Por otro lado, el apego con los pares explica un $22,2 \%$ la variabilidad de la calidad en las relaciones románticas. Conclusiones: Finalmente, el grado de calidad en las relaciones románticas logra explicar el 14\% de variabilidad en el autoconcepto de los adolescentes.
\end{abstract}

Palabras clave: Adolescencia, apego, autoconcepto, relaciones románticas.

\begin{abstract}
Objetive: The objective of the study was to determine the influence of attachment figures (mother, father and peers) and the quality of romantic relationships about selfconcept in students of both sexes between ages of 16 to 20 years old belonging to a private university in Lima. Methods: The design was not experimental, explanatory scope and cross-section. To that, three instruments were used: Attachment Inventory with Parents and Peers (AIPP), the Multidimensional Self Concept Scale Form 5 (SF5) and Quality Inventary in Romantic Relationships (NRI). Results: The results showed that attachment figures and the quality of romantic relationships can to explain the $44.7 \%$ of the variability in self-concept of adolescents. Also it was identified that the attachment figures can explain the $43.7 \%$ of the self-concept. Similarly, the family selfconcept explain in a $50.4 \%$ by attachment figures, being the mother the most influential figure. Continued to the result, it was identified a $25.9 \%$ of variability of social self-concept is explained by attachment with the peers. On the other hand, the attachment with peers explains a $22.2 \%$ the variability of the quality in romantic relationships. Conclusions: Finally, the quality level in romantic relationships can explain 14\% of variability in self-concept of teenagers.
\end{abstract}

Keywords: Adolescence, attachment, self-concept, romantic relationship.

\footnotetext{
${ }^{1}$ Psicóloga educativa, Colegio Jorge Chávez Dartnell, Huancavelica, Perú.

${ }^{2}$ Consultora - Free Lance. Consultora de Reclutamiento y Selección de Personal Selectiva, Lima, Perú.

${ }^{3}$ Profesora Asociada de Psicología. Directora de Acreditación. Facultad de Ciencias de la Salud. Universidad Peruana Unión, Lima, Perú.
} 


\section{INTRODUCCIÓN}

La adolescencia se caracteriza por ser una de las fases más fascinantes y quizá la más compleja, teniendo en cuenta que durante estos años el adolescente deja de recibir los cuidados que tenía en la niñez y aún no se ha consolidado las habilidades que le permitirá comportarse como adulto responsable, tal incertidumbre lo introduce en la búsqueda de autonomía, con la finalidad de consolidar su identidad, la cual se construye en base a tres pilares fundamentales: individual, familiar y social. De modo que su implicancia es de suma importancia sobre la formación del concepto que ha de adquirir el adolescente. Siendo así, el vínculo afectivo que establece con sus padres y pares, además de las experiencias de tipo romántico, son predictores que favorecen la construcción del autoconcepto (Sureda, 1998; Ros, Morandi, Cozzeti, Cornella y Surís, 2001; Penagos, Rodríguez, Carrillo y Castro, 2006; Esnaola, Goñi y Madariaga, 2008; Martínez, Castañeiras y Posada, 2011; Delgado, Oliva y Sánchez-Quejía, 2011; Martínez, Fuertes, Orgaz, Vicario y Gonzáles, 2014).

Asimismo, los sistemas donde interactúa el adolescente son diversos, siendo los principales el hogar y las instituciones académicas. Según el Instituto Nacional de Estadística e Informática (2010), dentro de Lima Metropolitana, hay un promedio de 32,9\% estudiantes menores de 20 años que ingresan a universidades privadas. Tales datos hacen referencia a aquellos adolescentes que, a través de una carrera universitaria, inician la búsqueda de independencia, crecimiento y madurez para el desarrollo de su autonomía y preparación a la adultez. Sin embargo, estas características podrían incrementarse si se considera que los estudiantes viven en internados o en residencias, lejos de su hogar, donde la relación parental puede verse disminuida por la distancia, dando mayor relevancia a las relaciones con sus pares.

Por lo expuesto, el estudio pretende evaluar la influencia del vínculo afectivo, con las figuras de apego y la calidad en las relaciones románticas sobre el autoconcepto, en estudiantes universitarios.

\section{Apego adolescente}

Una variable importante, en el estudio del autoconcepto, es el apego que es el vínculo afectivo creado en la infancia; la naturaleza y características de este, constituyen una de las bases y modelos sobre los cuales se desarrollará la percepción futura de uno mismo y la concepción personal de la relación con los demás, ya que es predictor para variables de satisfacción y equilibrio personal, a lo largo de la vida (Moneta, 2003).

Oliva (2011) sostiene que los distintos modelos de apego aprendidos, en la primera infancia, van a servir como predictor de gran influencia en el desarrollo de los procesos relacionales, es así que el adolescente resolverá mejor la tarea si consigue autonomía emocional de sus padres y un adecuado desempeño, en las relaciones amicales y románticas.

Allen y Miga (2008) definen al apego en la adolescencia como el período, donde este sistema puede ser considerado como una organización global, única e integrada a partir de la reflexión sobre las experiencias de las relacionales previas, las cuales ayudarán a mostrar estabilidad en el comportamiento del adolescente, dentro y fuera de su familia.

Ainsworth (1978) clasificó al apego como seguro, evitativo y ambivalente. Por su lado, Penagos, Rodríguez, Carrillo y Castro (2006), tras su investigación en adolescentes, proponen características básicas para cada modelo; donde el apego seguro está referido por niveles altos de confianza, cercanía y patrones adecuados de comunicación; el apego evitativo se distingue por la baja confianza, patrones inadecuados de comunicación y mínimos deseos de proximidad y cercanía; por último, el apego ambivalente se caracteriza por bajos niveles de confianza en el establecimiento de relaciones afectivas, deseo de mantener proximidad con estas, aunque todo esté acompañado de temor e inseguridad.

Esta etapa está caracterizada por diversos cambios, tanto físicos como psicológicos. Es esta línea Martínez, Castañeiras y Posada (2011) sostienen que, en la adolescencia, el apego sufre ciertas transformaciones entre las cuales está el hecho de que el adolescente no es solo receptor de cuidados, sino que también se vuelve un ente afectivo brindando apoyo a otros, estableciendo relaciones cada vez más simétricas con sus pares y/o relaciones amorosas.

\section{Calidad en las relaciones románticas en la adolescencia}

En la adolescencia el amor y la afectividad envuelve a toda la persona. La capacidad de amar, una ternura desbordante y cariño inmenso es invadido en esta etapa. Los chicos tienen diferentes maneras de amar que las chicas. En ellos reina el instinto sexual en cambio ellas se llenan de más afectividad y cariño. La atracción que se empieza a sentir se 

privada de Lima Este-Perú

manifiesta por el deseo de descubrir al otro como ser distinto pero complementario. Por esto, la afectividad es un encuentro gozoso entre dos personas que se atraen, se gustan y se quieren. Es sentida plenamente como el camino que conduce al amor utilizando la inteligencia, la madurez, los valores y la voluntad de querer realizarlo (Gonzáles, 2004).

Carrizo (2011), en su investigación, indica que el amor puede ser entendido como un triángulo (intimidad, pasión y compromiso), según la teoría del amor de Stemberg, por tal motivo decidió evaluar el predominio de las características del amor en dos etapas de la adolescencia: la adolescencia media y tardía. Los resultados indicaron que la característica que predomina en ambos grupos es la intimidad, explicado como la autoexposición, es decir, el mostrarse tal como es frente a su pareja. Seguidamente, el aspecto pasional y finalmente el compromiso.

En contrariedad, Vargas y Barrera (2002) comentan que el principal interés de los adolescentes al iniciar sus relaciones románticas, mayoritariamente se fundamenta en encontrar el concepto de sí mismo y la oportunidad de reafirmarse frente al grupo de los pares y los padres.

En apoyo, Silva (1997) menciona en su gran mayoría las pasiones, aventuras de connotaciones románticas son mecanismos mediante el cual pueden construir su identidad y ser reconocidos por sus pares.

\section{Autoconcepto adolescente}

El autoconcepto en la adolescencia se entiende como la forma en que cada adolescente se evalúa, se valora, teniendo en cuenta dos características importantes: confianza y seguridad en sí mismos. Por tal motivo, cumple un papel importante en los éxitos y fracasos, en sus relaciones interpersonales y metas (Urquijo, 2002).

Madrigales (2012) afirma que el grado en que el adolescente le dé interés a aspectos particulares de sí mismo y el aprecio, de sus habilidades en diferentes áreas de funcionamiento de su vida, serán el fundamento del autoconcepto, un constructo multidimensional.

Sureda (1998) añade que esta etapa tiene características peculiares, una serie de cambios físicos, sociales y psíquicos los que podrían actuar como perturbadores del propio concepto adolescente. Por tal motivo, propone cinco características que podrían relacionarse con el desarrollo identitario del adolescente, estos son: cambios fisiológicos, relaciones familiares, con pares del mismo y distinto sexo, desarrollo cognitivo e identidad personal.

\section{MATERIAL Y MÉTODOS}

Debido a las características de la muestra y al problema de investigación, el diseño es no experimental y de corte trasversal. Asimismo, es un estudio descriptivo explicativo, ya que se describió el comportamiento de cada una de las variables y se creó modelos para explicar la influencia del vínculo afectivo con las figuras de apego y la calidad en las relaciones románticas sobre el autoconcepto (Hernández, Fernández y Baptista, 2006).

La muestra estuvo conformada por 215 estudiantes de ambos sexos (femenino 54,4\% y masculino 45,6\%) entre las edades 16 a 20 años; donde el 33\% tiene 18 años, seguido por los 20 años $(29,8 \%)$ y solo el $0.5 \%$ tiene 16 años. Todos pertenecientes a una universidad privada de Lima de estrato económico bajo, medio y alto donde más de la mitad (78\%) se ubicaron en el nivel medio.

\section{Instrumentos}

\section{Inventario de Apego con Padres y Pares (IPPA)}

Creado por Armsden y Greenberg (1987), con el propósito de evaluar las puntuaciones del vínculo afectivo con las figuras de apego (madre, padre y pares) por medio de tres subdimensiones: confianza, comunicación y alienación. Adaptado al español por Penagos, Rodríguez, Carrillo y Castro en Colombia el año 2006. Está conformado por 75 ítems divididos en tres grupos de 25 afirmaciones. La calificación se da de acuerdo a la escala Likert de 5 puntos. Este instrumento ha demostrado tener una alta consistencia interna y fiabilidad, siendo aplicada en poblaciones peruanas en adolescentes. Específicamente para este estudio los índices de confiabilidad y validez de contenido obtenidos, para cada una de las figuras de apego, fueron: apego con madre $\alpha=, 895$; apego con padre $\alpha=, 927$ y apego con pares $\alpha=, 895$ y el apego total $\alpha=, 948$. Además, se obtuvo correlaciones altas $(\mathrm{p}<05)$ respecto a la validez de contenido del inventario total con sus figuras; madre $(\mathrm{r}=, 835)$, padre $(\mathrm{r}=, 812)$ y pares $(\mathrm{r}=706)$. 


\section{Cuestionario de Calidad de las Relaciones Románticas (NRI)}

Creada por Furman y Wehner (1994) tiene como fin evaluar la percepción de los estudiantes respecto a la calidad en su relación sentimental. Consta de 21 ítems repartidos en cuatro dimensiones; comunicación, compañía, expectativas del futuro y conflicto de formato de respuesta es de tipo Likert donde $1=$ nunca y $5=$ siempre. El índice de confiabilidad, obtenido en este estudio del cuestionario global, fue $\alpha=, 880$. Respecto a la validez de contenido de la escala global con sus dimensiones fue: comunicación $(\mathrm{r}=, 813$; $\mathrm{p}<, 05)$, compañía $(\mathrm{r}=, 779 ; \mathrm{p}<, 05)$, expectativas del futuro $(\mathrm{r}=, 779 ; \mathrm{p}<, 05)$ y conflicto $(\mathrm{r}=, 608 ; \mathrm{p}<, 05)$ y para la validez de constructo, el instrumento fue evaluado por tres jueces expertos, quienes aprobaron al $100 \%$ de relevancia, pertinencia y claridad.

\section{Escala Multidimensional de Autoconcepto Forma 5 (AF5)}

Creada por García y Musito (1999), consta de 30 ítems que evalúan el autoconcepto de manera global y de manera específica, en cinco dimensiones: académico, social, emocional, familiar y físico. El formato de respuesta es de tipo Likert donde $1=$ totalmente en desacuerdo y $5=$ totalmente de acuerdo. Este instrumento ha demostrado tener una alta confiabilidad del autoconcepto general $(\alpha=, 880)$ y sus dimensiones, como: académico $(\alpha=, 823)$, social ( $\alpha=, 763)$, emocional $(\alpha=, 800)$, familiar $(\alpha=, 743)$ y físico $(\alpha=753)$. Además, se obtuvo correlaciones altas $(\mathrm{p}<, 05)$ respecto a la validez de contenido del inventario total con sus dimensiones; académico $(\mathrm{r}=, 673)$, social $(\mathrm{r}=, 752)$, emocional $(\mathrm{r}=, 610)$, familiar $(\mathrm{r}=, 732)$ y familiar $(\mathrm{r}=, 641)$.

Para el análisis de los datos obtenidos, se utilizó el Paquete Estadístico para las Ciencias Sociales (SPSS) versión 22.00. Para contrastar las hipótesis planteadas, se determinó un nivel de significancia del 0,05 . Para evaluar el efecto de las variables, se utilizó el Estadístico de Regresión Lineal Múltiple donde se propuso modelos para explicar la influencia del vínculo afectivo con la figuras de apego, la calidad en las relaciones románticas, y el autoconcepto y sus dimensiones.

\section{RESULTADOS}

\section{Análisis descriptivo}

Se presentan los resultados descriptivos de las figuras de apego (padre, madre y pares), los niveles del autoconcepto y sus dimensiones, además del grado de calidad de relaciones románticas.

\section{Figuras de apego}

Se realiza el análisis descriptivo de las figuras de apego, respecto a sus medidas de tendencia central y variabilidad:

Tabla 1

Estadística descriptiva de las figuras de apego (padre, madre y pares) del estudiante

\begin{tabular}{lllllll}
\hline Instrumentos & Componentes & $\mathrm{M}$ & Mdn & SD & Mix. & Max. \\
\hline \multirow{2}{*}{$\begin{array}{l}\text { Inventario de apego } \\
\text { adolescente }\end{array}$} & Apego con la madre & 61,90 & 63 & 13,77 & 16 & 85 \\
& Apego con el padre & 54,04 & 57 & 15,73 & 6 & 84 \\
& Apego con los pares & 51,22 & 53 & 13,69 & 12 & 77 \\
\hline
\end{tabular}

En la tabla 1, se encontró que la muestra se caracteriza por tener puntuaciones que varían considerablemente, por tanto, se utilizará la mediana para describir el apego. Por lo tanto, los estudiantes resaltan medianas respecto a cada escala de apego con la madre $\mathrm{Mdn}=63$, con el padre $\mathrm{Mdn}=57$ $\mathrm{y} \mathrm{Mdn}=53$. 
Vínculo afectivo con las figuras de apego, calidad en las relaciones románticas y autoconcepto en estudiantes de una universidad privada de Lima Este-Perú

\section{Calidad de relaciones románticas}

Se describen los grados de la calidad de relaciones románticas.

\section{Tabla 2}

Niveles de calidad de relaciones románticas en los estudiantes

\begin{tabular}{lll}
\hline & $\mathrm{N}$ & $\%$ \\
\hline Deficiente calidad & 74 & $34,4 \%$ \\
Moderada calidad & 77 & $35,8 \%$ \\
Buena calidad & 64 & $29,8 \%$ \\
\hline
\end{tabular}

En la tabla 2 se evidencia que los estudiantes se caracterizan por mantener niveles moderados
$(35,8 \%)$ y deficientes $(34,4 \%)$, en referencia a la calidad en las relaciones románticas que mantienen.

\section{Autoconcepto}

Se realiza el análisis de los niveles de autoconcepto general y sus dimensiones:

\section{Tabla 3}

Niveles de autoconcepto general y sus dimensiones en estudiantes mujeres

\begin{tabular}{lllllll}
\hline & \multicolumn{2}{l}{ Bajo } & \multicolumn{2}{l}{ Moderado } & \multicolumn{2}{l}{ Alto } \\
\hline & $\mathrm{N}$ & $\%$ & $\mathrm{~N}$ & $\%$ & $\mathrm{~N}$ & $\%$ \\
\cline { 2 - 7 } Autoconcepto general & 45 & 20,9 & 80 & 37,2 & 90 & 41,9 \\
Autoconcepto académico & 31 & 14,4 & 60 & 27,9 & 124 & 57,7 \\
Autoconcepto emocional & 72 & 33,5 & 88 & 40,9 & 55 & 25,6 \\
Autoconcepto social & 53 & 24,7 & 89 & 41,4 & 73 & 34 \\
Autoconcepto familiar & 50 & 23,3 & 85 & 39,5 & 80 & 37,2 \\
Autoconcepto físico & 34 & 15,8 & 60 & 27,9 & 121 & 56,3 \\
\hline
\end{tabular}

En la tabla 3, se describe que las mujeres universitarias están caracterizadas por manifestar niveles elevados $(41,9 \%)$ y moderados $(37,2 \%)$ en el conocimiento de sí mismas. Respecto a sus dimensiones, la mayoría presenta niveles altos en cuanto a la dimensión académica $(57,7 \%)$ y física $(56,3 \%)$, sin embargo, un porcentaje considerable $(33,5 \%)$ manifiesta bajos niveles en el autoconcepto emocional.

Tabla 4

Niveles de autoconcepto general y dimensiones en estudiantes varones

\begin{tabular}{lllllll}
\hline & Bajo & \multicolumn{3}{l}{ Moderado } & Alto \\
\hline & $\mathrm{N}$ & $\%$ & $\mathrm{~N}$ & $\%$ & $\mathrm{~N}$ & $\%$ \\
\cline { 2 - 7 } Autoconcepto general & 33 & 15,3 & 120 & 55,8 & 62 & 28,8 \\
Autoconcepto académico & 18 & 8,4 & 73 & 34 & 124 & 57,7 \\
Autoconcepto emocional & 83 & 38,6 & 94 & 43,7 & 38 & 17,7 \\
Autoconcepto social & 43 & 20 & 78 & 36,6 & 94 & 43,7 \\
Autoconcepto familiar & 29 & 13,5 & 87 & 40,5 & 99 & 46 \\
Autoconcepto físico & 34 & 15,8 & 80 & 37,2 & 101 & 47 \\
\hline
\end{tabular}


En cuanto a los autoconcepto en los estudiantes varones, en la tabla 4 se expone que más de la mitad de los estudiantes varones $(55,8 \%)$ se caracterizan por mostrar niveles moderados en la percepción de sí mismos. En cuanto a sus dimensiones; la mayoría manifiesta elevados puntajes en la dimensión académica $(57,7 \%)$, social $(43,7 \%)$, familiar $(46 \%)$ y física (47\%), a diferencia de la dimensión emocional, que al igual que la muestra femenina, un porcentaje considerable evidencia niveles bajos $(38,6 \%)$.

\section{Análisis de regresión}

\begin{tabular}{lllllll}
\hline Regresión & R & R2 & Gl & F & p & VIF \\
\hline Modelo 1*autoconcepto &, 679 &, 447 & 4 & 45,001 &, 000 & \\
Apego con madre & & & & &, 000 & 1,712 \\
Apego con padre & & & & &, 000 & 1,638 \\
Apego con pares & & & & &, 000 & 1,472 \\
Calidad de relaciones románticas & & & & &, 031 & 1,326 \\
\hline
\end{tabular}

Se realizan los análisis de regresión, en los cuales se proponen 10 modelos que explican el efecto de las variables independientes, frente a la dependiente.

Tabla 5

Influencia de las figuras de apego y la calidad en las relaciones románticas, en el autoconcepto de estudiantes Modelo 1: Autoconcepto (variable dependiente)

\begin{tabular}{|c|c|c|c|c|c|c|}
\hline Regresión & $\mathrm{R}$ & R2 & $\mathrm{gl}$ & $\mathrm{F}$ & $p$ & VIF \\
\hline Modelo 2*autoconcepto & ,667 & ,437 & 3 & 56,377 & 000 & \\
\hline Apego con madre & & & & & 000 & 1,63 \\
\hline Apego con padre & & & & & ,000 & 1,48 \\
\hline Apego con pares & & & & & 000 & 1,21 \\
\hline Modelo $3^{*}$ Autoconcepto familiar & 715 & ,504 & 3 & 73,546 & 000 & \\
\hline Apego con madre & & & & &, 000 & 1,63 \\
\hline Apego con padre & & & & & ,004 & 1,48 \\
\hline Apego con pares & & & & & ,002 & 1,21 \\
\hline Modelo $4^{\star}$ Autoconcepto emocional & ,439 & ,181 & 3 & 16,809 &, 000 & \\
\hline Apego con madre & & & & & 092 & 1,63 \\
\hline Apego con padre & & & & & ,224 & 1,48 \\
\hline Apego con pares & & & & & ,000 & 1,21 \\
\hline Modelo 5* Autoconcepto social & ,519 & 259 & 3 & 25,966 &, 000 & \\
\hline Apego con madre & & & & & ,226 & 1,63 \\
\hline Apego con padre & & & & & ,598 & 1,48 \\
\hline Apego con pares & & & & & ,000 & 1,21 \\
\hline Modelo 6* Autoconcepto académico & ,395 & ,144 & 3 & 12,992 &, 000 & \\
\hline Apego con madre & & & & & ,292 & 1,63 \\
\hline Apego con padre & & & & & ,003 & 1,48 \\
\hline Apego con pares & & & & & 009 & 1,21 \\
\hline Modelo $7^{\star}$ Autoconcepto físico & ,397 & , 146 & 3 & 13,198 & ,000 & \\
\hline Apego con madre & & & & & ,119 & 1,63 \\
\hline Apego con padre & & & & & 000 & 1,48 \\
\hline Apego con pares & & & & & ,560 & 1,21 \\
\hline
\end{tabular}



privada de Lima Este-Perú

En la tabla 5, se aprecia que el vínculo afectivo, con las figuras de apego (madre, padre y pares) y la calidad en las relaciones románticas, logran explicar el $44,7 \%$ del nivel de autoconcepto de los adolescentes ( $\mathrm{f}=45,001 ; \mathrm{p}<, 05)$. Además, se observa que el coeficiente de inflación de la varianza (VIF) de las variables independientes tienen valores cercanos a 1 , lo que indica la no multicolinealidad entre ellas.

\section{Tabla 6}

Influencia de las figuras de apego respecto al autoconcepto y sus dimensiones

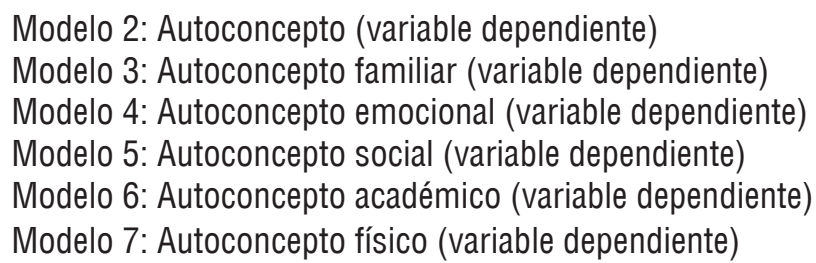

En la tabla 6, se aprecia que el vínculo afectivo, con las figuras de apego, explican el nivel del autoconcepto en un $43,7 \%$, siendo las tres variables relevantes en la predicción $(\mathrm{f}=56,377 ; \mathrm{p}<, 05)$. En cuanto a las dimensiones, se evidencia que el nivel de autoconcepto familiar es explicado en un 50,4\%, por el efecto de los vínculos que se mantiene con las figuras de apego $(\mathrm{f}=73,546 ; \mathrm{p}<, 05)$. Respecto al autoconcepto social, se observa que un $25,9 \%$ se explica por el vínculo afectivo de apego con los pares $(\mathrm{f}=25,966 ; \mathrm{p}<, 05)$. Por otro lado, solo el apego con los pares es el que explica la variabilidad del autoconcepto emocional solo en un $18,1 \%$ ( $\mathrm{f}=16,809$; $\mathrm{p}<, 05)$. Finalmente, los demás modelos no son relevantes para explicar la influencia de la dimensión académica y física. Adicionalmente, se aprecia que el coeficiente de inflación de la varianza (VIF) de las variables independientes tienen valores cercanos a 1 , lo que indica la no multicolinealidad entre las variables.

Tabla 7

Influencia de las figuras de apego respecto a la calidad de relaciones románticas

Modelo 8: Calidad en las relaciones románticas (variable dependiente) Modelo 9: Calidad en relaciones románticas (variable dependiente)

\begin{tabular}{lllllll}
\hline Regresión & $\mathrm{R}$ & $\mathrm{R} 2$ & $\mathrm{Gl}$ & $\mathrm{F}$ & $\mathrm{P}$ & $\mathrm{VIF}$ \\
\hline Modelo 8*${ }^{*}$ calidad &, 486 &, 226 & 3 & 21,772 &, 000 & \\
Apego con madre & & & & &, 896 & 1,63 \\
Apego con padre & & & & &, 077 & 1,48 \\
Apego con pares & & & & &, 000 & 1,22 \\
Modelo 9* apego con pares &, 471 &, 222 & 1 & 60,756 &, 000 & 1 \\
\hline
\end{tabular}

En cuanto al efecto de las figuras de apego en la calidad de las relaciones románticas en los estudiantes, en la tabla 7 se aprecia un $22,6 \%$ de influencia en la calidad de relaciones románticas frente al vínculo de las figuras de apego, donde el apego con pares es más relevante para explicar el modelo $(\mathrm{p}<0,05)$, siendo la única variable independiente significativa. Además se evidencia que el coeficiente de inflación de la varianza (VIF) de las variables independientes tiene valores cercanos a 1 , lo que indica la no multicolinealidad entre las variables. 
Tabla 8

Influencia del autoconcepto en la calidad de relaciones románticas

\begin{tabular}{lllllll}
\hline Regresión & $\mathrm{R}$ & $\mathrm{R} 2$ & $\mathrm{gl}$ & $\mathrm{F}$ & $\mathrm{p}$ & $\mathrm{VIF}$ \\
\hline $\begin{array}{l}\text { Model010* autoconcepto } \\
\text { Calidad }\end{array}$ & & & & & & \\
\hline
\end{tabular}

En la tabla 8, se aprecia que la calidad en las relaciones románticas logran explicar el 14,4\% el nivel de autoconcepto de los adolescentes ( $\mathrm{f}=35,767$; $\mathrm{p}<, 05)$. Se aprecia además que el coeficiente de inflación de la varianza (VIF) de las variables independientes tienen valores cercanos a 1, lo que indica la no multicolinealidad entre las variables.

\section{DISCUSIÓN}

El objetivo de esta investigación fue determinar el grado del efecto de los vínculos afectivos con las figuras de apego (madre, padre y pares), y la calidad en las relaciones románticas sobre el nivel de autoconcepto.

De manera global, la mayoría de estudiantes evaluados muestran puntajes adecuados respecto a sus figuras de apego; por lo tanto, se afirma que en la adolescencia, además de mantener el vínculo afectivo con los cuidadores primarios, se consolida el vínculo con los pares. De manera similar Lieberman, Doyle y Markiewicz (1999) señalan que la disponibilidad, como la confianza con los padres, siguen siendo importantes en la infancia y adolescencia; sin embargo, lo que disminuye con la edad es la dependencia hacia los padres. A esto Nieves (2009) agrega que el adolescente ha adquirido mayor capacidad mental y física, por lo que la necesidad de proximidad y la protección en situaciones de amenaza o tensión se reduce. No obstante Delgado, Oliva y Sánchez (2011) sostienen que aún son importantes la comunicación (grado de calidad de la comunicación verbal), confianza (comprensión y respeto en las relaciones) y cercanía de sus cuidadores.

En cuanto a la calidad de relaciones románticas, se observó que la mayoría de los evaluados puntúan dentro de los niveles moderados y bajos. Rivera, Cruz y Muñoz (2011) realizaron un estudio donde uno de sus objetivos fue describir la satisfacción con la relación de pareja, en una muestra de personas entre los 18 y 26 años, se concluyó que las relaciones satisfactorias son predominantes en parejas con mayor estabilidad y compromiso, y se dan frecuentemente a medida que se acercan al término de la adultez emergente. De modo que al analizar la muestra del presente estudio, aún se encuentran en la etapa de transición entre las relaciones amicales y los compromisos formales propios del adulto, resaltando en ellos las relaciones fugaces de escasa intimidad, basadas mayormente en la atracción sexual, compañía, afecto, reciprocidad y reafirmación social. Por su parte, White (1967) refiere que las uniones románticas, antes de los 20 años, frecuentemente han resultado en separaciones deshonrosas, ya que carecen de experiencia, madurez, criterio y compromiso.

Respecto al autoconcepto en los estudiantes, Musitu, García y Gutiérrez (1997) consideran que es una estructura multidimensional y jerárquica, en apoyo Markus y Wurf (1987); Díaz, Prados y López (2002) y Esnaola, Goñi y Madariaga (2008) adhieren que cada dominio personal es diferente, lo cual hace que la percepción sea distinta en cada área de comportamiento.

Por ello, se realizó un análisis dimensional, donde la muestra femenina obtuvo nivel alto en el autoconcepto general, académico y físico; además puntaje moderado en las dimensiones social y familiar. En el caso de los varones, se caracterizan por obtener un nivel moderado respecto al autoconcepto general, mientras que en el autoconcepto social, familiar y físico obtuvieron un puntaje alto. Llama la atención que en el autoconcepto emocional ambos se ubicaron en niveles moderados y bajos, lo cual podría indicar que en la actualidad los estudiantes manifiestan una disminución en la autopercepción del equilibrio emocional, compromiso, seguridad y confianza en sus recursos para asumir situaciones que les generan ansiedad. Resultados similares obtuvieron Penagos, Rodríguez, Carrillo y Castro (2006), donde encontraron que la mayoría de adolescentes mantienen un autoconcepto moderado, infiriendo que se encuentran en incertidumbre por la búsqueda de identidad; sin embargo, en el intento de autoprotección, suelen mostrarse seguros y fuertes.

Respecto a la influencia del vínculo afectivo, con las figuras de apego y la calidad en las relaciones románticas sobre el autoconcepto, se encontró que ambas variables logran explicar el $44,7 \%$ del grado en 
cómo se perciben los adolescentes ( $\mathrm{f}=45,001, \mathrm{p}<0.05$ ). Entonces, el autoconcepto se construye a lo largo de vida por las experiencias de éxito o fracaso y la interacción con personas significativas. Como señalan Sureda (1998) y Arpini y Quintana (2002), la formación de la percepción, de sí mismo, es producto de diversas interacciones como el cambio fisiológico, la relación familiar, con los pares (del mismo y del sexo opuesto), desarrollo cognitivo y la autoimagen. Entonces, el modelo propuesto refleja y reúne lo que diversos investigadores (Del Río, Barrera y Vargas, 2004; Gómez, Pérez, Vargas y Wilthew, 2010; Martínez, Castañeiras y Posada, 2011; Martínez, Fuertes, Orgaz, Vicario y Gonzáles, 2014) han señalado sobre la influencia de la calidad en las relaciones románticas y el vínculo afectivo sobre el autoconcepto de los adolescentes, siendo esta una interacción mutua, (Slotter, Gardner y Finkel, 2010).

El siguiente modelo explica el efecto del vínculo afectivo con las figuras de apego, en un $43,7 \%$, sobre el nivel de autoconcepto. Resultados similares obtuvieron Penagos, Rodríguez, Carrillo y Castro (2006), quienes concluyen que la percepción de los adolescentes, respecto al apego con sus padres y pares, ejerce una influencia importante en el nivel de autoconcepto. Por lo tanto, podemos inferir que casi la mitad del constructo es explicado solamente por los vínculos afectivos, tanto con los cuidadores primarios como con los pares, siguiendo esta línea Moreno (2010) afirma que el apego crea bases que consolidarán las habilidades de relacionamiento cercano y, por ende, la construcción de la percepción de sí mismo.

Asimismo, al analizar las dimensiones del autoconcepto, se encontró que la dimensión familiar es influida en un 50,4\%, por el vínculo con las figuras de apego $(\mathrm{f}=56,377 ; \mathrm{p}=0,05)$. Propuesta apoyada por Adell, quien sostiene que la familia es el seno donde se establecen los primeros aprendizajes sociales, donde se conforman las pautas de comportamiento y se inicia el desarrollo de la personalidad (citado por Salum, Marín y Reyes, 2011). Respecto al autoconcepto social, se aprecia que solamente el vínculo afectivo con los pares explica el 25,9\% ( $\mathrm{f}=25,966 ; \mathrm{p}=0,05)$, ya que el vínculo afectivo con los padres no es un factor importante para explicar esta dimensión. En concordancia con los resultados Sureda (1998), Paz y Serra (2009) reportan que el autoconcepto adolescente se ve influenciado, en gran manera, por agentes sociales (pares de igual y distinto sexo), los cuales aportan a la popularidad, aceptación e integración; estos son logros indispensables para su desarrollo personal. Asimismo, el vínculo con los pares cobra importancia al explicar al autoconcepto emocional
$18,1 \% \quad(\mathrm{f}=16,809 ; \mathrm{p}=0,05)$. Sureda (1998) indica la importancia de los compañeros, como fuente de comprensión y apoyo en la reafirmación personal, ya que si la relación es amigable e íntima, harán un mejor uso de estrategias positivas y constructivas en la disminución de conflictos. Cabe considerar que en este estudio los universitarios se encuentran alejados físicamente del hogar; situación que ayuda al proceso de independización $\mathrm{y}$, por ende, a vincularse más con las figuras cercanas (pares), convirtiéndolos en su principal soporte. No obstante, esto no ocurre con el autoconcepto académico $(\mathrm{f}=12,992 ; \mathrm{p}=0,05) \quad y$ físico $(\mathrm{f}=13,198 ; \mathrm{p}=0,05)$ quienes son explicados solo en un 14\%, lo que puede indicar que su variabilidad puede ser determinada por otros constructos. De manera análoga, en cuanto al autoconcepto académico Salum, Marín y Reyes (2011) asumen que el adolescente es capaz de tener en cuenta múltiples criterios para juzgar su capacidad académica, dentro de los cuales se encuentra su rendimiento académico, reconocimiento del docente, comparación con sus compañeros, esfuerzo empleado, dificultad de las tareas y la crítica o aceptación de su desempeño. Respecto al autoconcepto físico Rodríguez, Goñi y Ruiz de Azúa (2006) llevaron a cabo un estudio donde concluyeron que para su constitución es importante el estilo de vida y el bienestar psicológico, sin embargo, consideran que las variables individuales (personalidad, valores, ideas, percepción, etc.) y contextuales (familia, grupo de amigos, medios de comunicación y sociedad) también pueden ejercer una influencia considerable.

Por otro lado, datos interesantes se encontraron al evaluar la influencia del vínculo afectivo, con los figuras de apego sobre la calidad en las relaciones románticas, ya que las figuras parentales (madre, padre) no eran significativas para explicar el modelo $(p<0,896 ; p<0,077)$ caso contrario, el vínculo afectivo con los pares ejerció un efecto del $22 \%(\mathrm{f}=60,756 ; \mathrm{p}<0,05)$, explicando así el modelo completo. Debido a que el adolescente se sentirá más seguro y confiado al afrontar situaciones nuevas, si lo hace en compañía de un buen amigo, ya que una amistad adecuada preparará el camino para el comienzo de relaciones en pareja (Oliva, 2011). Pero a la vez se aprecia que hay un porcentaje mayoritario que puede verse explicado por otras variables como la atracción física, características de personalidad, compatibilidad de interés o habilidades y los valores personales, religiosos y culturales (Brown, Feiring y Furman, 1999).

Finalmente, la calidad en las relaciones románticas explicó, en un $14 \%$, los niveles de autoconcepto. Propuesta corroborado en el estudio de Slotter, Gardner 
y Finkel (2010), quienes analizaron la variabilidad del autoconcepto tras una ruptura amorosa en universitarios y encontraron que, después de una separación los miembros, tienden a reducir en contenido y claridad la percepción de sí mismos, ya que se produce un intenso dolor emocional que los lleva a redefinir individualmente lo que cada uno es, en ausencia del otro. Por ello, se puede inferir que, durante una relación romántica, el autoconcepto individual se entrelaza y las líneas que los diferencian se vuelven borrosas, dando una mayor centralidad a la relación en su vida. Por otro lado, se aprecia que los participantes de este estudio difieren en el grado de influencia del autoconcepto (14\%); por lo tanto, se infiere que las figuras de apego explican mejor su formación (43,7\%).

Se concluye que los estudiantes obtienen puntajes adecuados en cada escala, donde el apego con la madre mantiene una mediana de 63; el apego con el padre, de 57 y el apego con los pares, de 53. Por ello, se considera que la mayoría de adolescentes obtuvieron un estilo seguro de apego, respecto a las figuras estudiadas, aportando a los estudios que defienden la importancia de este vínculo a lo largo de la vida.

En cuanto a la calidad de las relaciones románticas en base a la confianza, comunicación, expectativas frente al futuro y ajuste al conflicto, los resultados encontrados indican que los de estudiantes las perciben como calidad baja y regular.

Respecto al autoconcepto, trabajado de manera multidimensional, jerárquico y por género, se evidenció que las mujeres mantienen niveles altos en el conocimiento general de sí mismas, explicado en su mayoría por las dimensiones académica y física, seguida de un puntaje moderado en el autoconcepto social y familiar $\mathrm{y}$, por último, con un nivel entre moderado y bajo en la dimensión emocional.

En cuanto a los varones, se expone que más de la mitad obtuvieron una puntuación moderada en el autoconcepto general, asimismo, las dimensiones académica, social, familiar y física mantienen niveles altos, a diferencia de la dimensión emocional que, al igual que las mujeres, se encuentran en niveles entre moderados y bajos.

Por otro lado, se encontró que la influencia del vínculo afectivo con las figuras del apego y la calidad en las relaciones románticas logran explicar, en un $44,7 \%$, el nivel de autoconcepto en los estudiantes.

Se encontró que el nivel afectivo, con las figuras de apego, ejerce un efecto de $43,7 \%$ sobre el autoconcepto general. Con respecto a las dimensiones del autoconcepto, se encontró que el vínculo afectivo con las figuras de apego explica, en un $50,4 \%$, la variabilidad del autoconcepto familiar; sin embargo, para las dimensiones del autoconcepto social y emocional, el vínculo afectivo con los pares explica el modelo en un $25,9 \%$ y $18,1 \%$ respectivamente.

En cuanto al efecto del vínculo afectivo, con las figuras de apego sobre la calidad en las relaciones románticas, es del 22\% siendo solo el apego con los pares el que predice el modelo total. Por último, la calidad en las relaciones románticas logra explicar un $14 \%$ de influencia sobre el nivel del autoconcepto.

Declaración de financiamiento y de conflicto de intereses:

El estudio fue financiado por los autores, quienes declaran no tener algún tipo de conflicto de interés en la investigación realizada.

\section{Correspondencia:}

\section{Yesenia Ávila Bacilio}

Psicólogo Consultor - Free Lance. Consultora de Reclumiento y Selección de Personal Selectiva. Calle Ramón Ribeyro 1095, Barranco. Lima. Perú.

e-mail: yavila.psic@gmail.com 


\section{REFERENCIAS BIBLIOGRÁFICAS}

Ainsworth, M. (1989). Attachments beyond infancy. American Psychologist. 44(4), 709-716. Recuperado de http://psycnet.apa.org/index.cfm?fa=buy. optionToBuy\&id=1989-25551-001.

Allen, J. \& Miga, E. (2010). Attachment in adolescence: $A$ move to the level of emotion regulation. NIHPA Manuscripts. 27 (2), 181-190. Recuperado de http:// www.ncbi.nlm.nih.gov/pmc/articles/PMC2860752/.

ANAR Perú. (2006). Dificultades en las relaciones interpersonales en adolescentes. El caso del teléfono ANAR. Recuperado de http://www.anarperu.org/ wpcontent/uploads/2013/06/200501-200612_ Relaciones_interpersonales_investigacion.pdf.

Armsden, G. \& Greenberg, M. (1987). The inventory of parent and peer attachment: Individual differences and their relationship to psychological well -being in adolescence. Journal of Youth and Adolescence, 16(5), 427-454. Recuperado de http://link.springer. com/article/10.1007\%2FBF02202939.

Armsden, G. \& Greenberg, M. (1987). The inventory of parent and peer attachment: Individual differences and their relationship to psychological well -being in adolescence. Journal of Youth and Adolescence. 16(5), 427-454. Recuperado de http://link.springer. com/article/10.1007\%2FBF02202939.

Arpini, D. \& Quintana, A. (2003). Identidade, família e relações sociais em adolescentes de grupos populares. Revista de Estudios de Psicologia, PUC Campinas. 20(1), 27-36. Recuperado de http://www. scielo.br/scielo.php?script=sci_arttext\&pid=s0103$166 \times 2003000100003$.

Brown, B., Feiring, C. \& Furman, W. (1999). Missing the love boat why researchers have shied away from adolescent romance. The development of romantic relationships in adolescence (1-16). New York: Cambridge University Press. Recuperado de https://www.du.edu/psychology/relationshipcenter/ publications/brown_feiring furman_1999.pdf.

Carrizo, S. (2011). Adolescencia y estilos de amor. (Tesis de Licenciatura), Universidad Abierta Interamericana, Argentina. Recuperado de www.imgbiblio.vaneduc. edu.ar/fulltext/files/TC104098.pdf.

Delgado, I., Oliva, A. \& Sánchez-Quejía, I. (2011). Apego a los iguales durante la adolescencia y la adultez emergente. Anales de la psicología, 27(1), 155-163. Recuperado de http://revistas.um.es/analesps/ article/download/113581/107571.

Díaz, F., Prados, M. \& López, S. (2002). Relación entre rendimiento académico, sintomas depresivos, edad y género. Psiquiatría.com, 6(2). Recuperado de http://hdl.handle.net/10401/1249.

Esnaola, I., Goñi, A. \& Madariaga, J. (2008). El autoconcepto: perspectivas de investigación. Revista de psicodidáctica, 13(1), 69-96. Recuperado de http://www.redalyc.org/articulo.oa?id=17513105.
Furman, W. \& Wehner, E.A. (1994). Romantic views: Toward a theory of adolescenct romantic relationships. California: Sage.

Furman, W. \& Buhrmenster, D. (2009). The Network of Relationships Inventory: Behavioral Systems Version. NIHPA Manuscripts. 33(5), 470-478. Recuperado de http://www.ncbi.nlm.nih.gov/pmc/articles/ PMC2826206/.

García, F. \& Musitu, G. (1999). AF5: Autoconcepto Forma 5. Madrid: TEA Ediciones.

González, J. (2004). Relaciones interpersonales. México: El Manual Moderno.

Hernández, R., Fernández, C. \& Baptista, P. (2006). Metodología de la investigación. cuarta edición. Ciudad de México: McGraw Hill/Interamericana Editores S.A. Recuperado de https://competenciashg. files.wordpress.com/.../sampieri-et-al metodologia-dela-investigacion-4ta-edicion-sampieri-2006_ocr.pdf.

Instituto Nacional de Estadística e Informática. (2010). Día mundial de la población. Recuperado de http://www. inei.gob.pe/media/MenuRecursivo/publicaciones digitales/Est/Lib1095/libro.pdf.

Lieberman, M., Doyle, A. \& Markiewicz, D. (1999). Developmental patterns in security of attachment to mother and father in late childhood and early adolescence: associations with peer relations. Child Dev, 70(1), 202-213. Recuperado de http://www.ncbi. nlm.nih.gov/pubmed/10191523.

Madrigales, C. (2012). Autoconcepto en Adolescentes de 14 a 18 años (estudio realizado en Funda Niñas Mazatenango). (Tesis de licenciatura), Universidad Rafael Landívar Quetzaltenango, México. Recuperado de www.biblio3.url.edu.gt/Tesis/2012/05/42/ Madrigales-Ceily.pdf.

Markus, H. \& Wurf, E. (1987). The Dynamic SelfConcept: A Social Psychological Perspective. Annual Review of Psychology, 38, 299-337. Recuperado de http://www.annualreviews.org/doi/abs/10.1146/ annurev.ps.38.020187.001503.

Martínez, J., Fuertes, A., Orgaz, B., Vicario, I. \& Gonzáles, E. (2014). Vínculos afectivos en la infancia y calidad en las relaciones de pareja de jóvenes adultos: el efecto mediador del apego actual. Anales de la Psicología. 30(1), 211-220. Recuperado de http://revistas.um.es/ analesps/article/view/analesps.30.1.135051.

Martínez, V., Castañeiras, C. \& Posada, C. (2011). Estilos de apego y autoconcepto: bases para una adolescencia positiva. Psicodebate. Psicología, Cultura y Sociedad, 11(1), 27-40. Recuperado de www.palermo.edu/ cienciassociales/publicaciones/pdf/.../11Psico_02.pdf .

Moneta, M. (2003). El apego: aspectos clínicos y psicobiológicos de la díada madre-hijo. Santiago de Chile: Cuatro Vientos. 
Moreno, R. (2010). Estilos de Apego en el Profesorado y Percepción de sus Relaciones con el Alumnado. (Tesis de doctorado), Universidad Complutense de Madrid, España. Recuperado de http://eprints.ucm. es/11580/1/T32256.pdf.

Nieves, M. (2009). Propiedades Psicométricas del Inventario de Apego con Padres y Pares (IPPA) en una muestra de adolescentes venezolanos. (Tesis de maestría), Universidad Rafael Urdaneta, Venezuela. Recuperado de www.200.35.84.131/portal/bases/marc/ texto/9214-09-02829.pdf.

Oliva, A. (2011). Apego en la adolescencia. Acción psicológica, 8(2), 55-65. Recuperado de http:// personal.us.es/oliva/apego $\% 20$ en $\% 201$ a $\% 20$ adolescencia.pdf.

Paz, M. \& Serra, E. (2009). Las relaciones de amor en los adolescentes de hoy. Barcelona: Octaedro. Recuperado de http://www.octaedro.com/downloadf. asp? $=10043$.pdf.

Penagos, A., Rodríguez, M., Carrillo, S. \& Castro, J. (2006). Apego, relaciones románticas y autoconcepto en adolescentes bogotanos. Universitas Psychologica, 5(001), 1-17. Recuperado de: http://web.ebscohost. com/ehost/pdfviewer/pdfviewer?sid=fc786aab-eb2e426b-8378-a5a141c8c4d3\%40sessionmgr 13\&vid=2\& hid=19.

Rivera, D., Cruz, C. \& Muñoz, C. (2011). Satisfacción en las relaciones de pareja en la adultez emergente: el rol del apego, la intimidad y la depresión. Terapia Psicológica. 29(1), 77-83. Recuperado de www. scielo.cl/pdf/terpsicol/v29n1/art08.pdf.

Rodríguez, A., Goñi, A. \& Ruiz de Azua, S. (2006). Autoconcepto físico y estilos de vida en la adolescencia. Intervención psicosocial, 15(1), 81-94. Recuperado de http://scielo.isciii.es/scielo.php?pid=S113205592006000100006\&script=sci_arttext
Ros, R., Morandi, T., Cozzeti, E., Cornella, J. \& Surís, J. (2001). Manual de salud reproductiva en la adolescencia. España: INO Reproducciones S.A.

Salum, A., Marín, R. \& Reyes, C. (2011). Autoconcepto y rendimiento académico en estudiantes de escuelas secundarias públicas y privadas de Ciudad Victoria, Tamaulipas, México. Revista Internacional de Ciencias Sociales y Humanidades, SOCIOTAM, 21(1), 207-229. Recuperado de http://www.redalyc. org/articulo.oa? $\mathrm{id}=65421407010$.

Silva, M. (1997). Sexualidad y adolescencia. Tópicos en psicología. Santiago: Impresos Universitaria, S.A.

Slotter, E., Gardner, W. \& Finkel, E. (2010). Who am I without you? The influence of romantic breakup on the selfconcept. Running Head, 36(2), 147-160. Recuperado de http://psp.sagepub.com/content/36/2/147.short.

Sureda, I. (1998). Autoconcepto y adolescencia: una línea de intervención psicoeducativa. Revista Mallorquina de Pedagogía, 11, 157-170. Recuperado de http:// dialnet.unirioja.es/servlet/articulo?codigo $=91224$.

Urquijo, S. (2002). Auto-concepto y desempeño académico en adolescentes. Relaciones con sexo, edad e institución. Psico-USF, 7(2), 211-218. Recuperado de www.scielo. br/pdf/pusf/v7n2/v7n2a10.pdf.

Vargas, E. \& Barrera, F. (2002). Adolescencia, relaciones románticas y actividad sexual: una revisión. Revista Colombiana de Psicología, 11, 115-134.

White, E. (1967). Mensajes para los jóvenes enamorados. Buenos Aires: Asociación Casa Editora Sudamericana.

Recibido: 19/01/2015 Aceptado: 28/04/2015 